\title{
A Ratiometric Quantum Dot-Ligand System Made by Phase Transfer for Visual Detection of Double-Stranded DNA and Single Nucleotide Polymorphism
}

\author{
Yuqian Liu, ${ }^{\dagger}$ Mingfu Ye, ${ }^{\dagger}$ Qinyu Ge, ${ }^{\ddagger}$ Xiaojun $\mathrm{Qu}^{\dagger}{ }^{\dagger}$ Qingsheng Guo, ${ }^{\dagger}$ Xianyun $\mathrm{Hu}^{\dagger}$ and Qingjiang Sun ${ }^{\dagger}$ \\ State Key Laboratory of Bioelectronics, School of Biological Science \& Medical Engineering, and Research Center for \\ Learning Science, Southeast University, Nanjing 210096, P. R. China
}

*Fax: (86)25-83792349; Email: sunqj@seu.edu.cn

\section{Contents:}

Table S1. DNA sequences used in the hybridization-based DNA assay.

Figure S1. FT-IR spectra of the QDs nanohybrid, dppz, QD-L sensor in the absence/presence of $30 \mathrm{mg} / \mathrm{L}$ ssDNA or ctDNA.

Figure S2. (A) Fluorescence spectra of 5-batches of rQD@SiO $@ g Q D$ nanohybrids (circle) and the corresponding QD-L sensors (diamond). (B) Relative emission intensity ratio of the QDs nanohybrid and the QD-L sensor. The inset shows fluorescent images of the QDs nanohybrid and the QD-L sensor for 5 batches (a-e).

Figure S3. DLS measurements showing size distribution of $\mathrm{rQD} @ \mathrm{SiO}_{2} @ g Q D$ nanoparticles in pure water, after phase transfer and in Tris- $\mathrm{HCl}$ buffer.

Figure S4. Ratiometric fluorescent response of the QD-dye ionic conjugate as well as the QD-L sensor versus time at different ctDNA concentrations.

Figure S5. (A) Fluorescence spectra of the rQD@SiO $@ g Q D:\left[R u(b p y)_{2}(d p p z)\right]^{2+}$ sensor in the presence of o, o.2, o.5, 1, 2, 5, 10, $30 \mathrm{mg} / \mathrm{L}$ ctDNA. The inset shows fluorescent images of this sensor at different concentrations of ctDNA. (B) Evolution of relative emission intensity ratio of the $\mathrm{rQD} @ \mathrm{SiO}_{2} @ \mathrm{gQD}:\left[\mathrm{Ru}(\mathrm{bpy})_{2}(\mathrm{dppz})\right]^{2+}$ sensor with respect to the ctDNA concentration.

Figure S6. Fluorescence spectra of the QD-L sensor and the QD-dye ionic conjugate in the presence of different biomolecules.

Figure S7. Molecular structures of (A) dppz and $\left[\mathrm{Ru}(\mathrm{bpy})_{2}(\mathrm{dppz})\right]^{2+}$, and (B) dsDNA, ssDNA, RNA and BSA.

Figure S8. Comparison of fabrication process between the QD-MB sensor and the QD-L sensor.

Figure S9. (A) Fluorescence spectra of the QD-MB sensor upon hybridizing with o, 0.2, 0.5, 1, 2, 3, 4, $5 \mu \mathrm{M}$ WT. (B) Fluorescence spectra of the QD-L sensor in the presence of the capture DNA $(5 \mu \mathrm{M})$ upon hybridizing with o, 0.2, $0.5,1,2,3$, 4, $5 \mu \mathrm{M}$ WT. (C) Evolution of relative emission intensity ratios of the sensors with respect to the concentrations of WT. The inset shows fluorescent images of the QD-L sensor and the QD-MB sensor at different WT concentrations, respectively.

Figure S1o. Fluorescence spectra of the QD-L sensor (in the presence of $3 \mu \mathrm{M}$ capture DNA) and the QD-MB sensor upon hybridizing with $3 \mu \mathrm{M} \mathrm{NC}, 1 \mathrm{BM}$ and WT. 
Table S1. DNA sequences used in the hybridization-based DNA assay.

\begin{tabular}{ll}
\hline Name & Sequence (5'-3') \\
\hline Capture DNA & TATGAAATCGGCTCCCGCAG \\
Molecular beacon (MB) & NH $_{2}$-ACGCAATATGAAATCGGCTCCCGCAGTTGCGT-BHQ1 \\
Wild type DNA (WT) & CTGCGGGAGCCGATTTCATA \\
1 base muted DNA (1BM) & CTGCGGGAGTCGATTTCATA \\
Noncomplementary DNA (NC) & GGCATCACTGGACAGCATCG \\
\hline
\end{tabular}

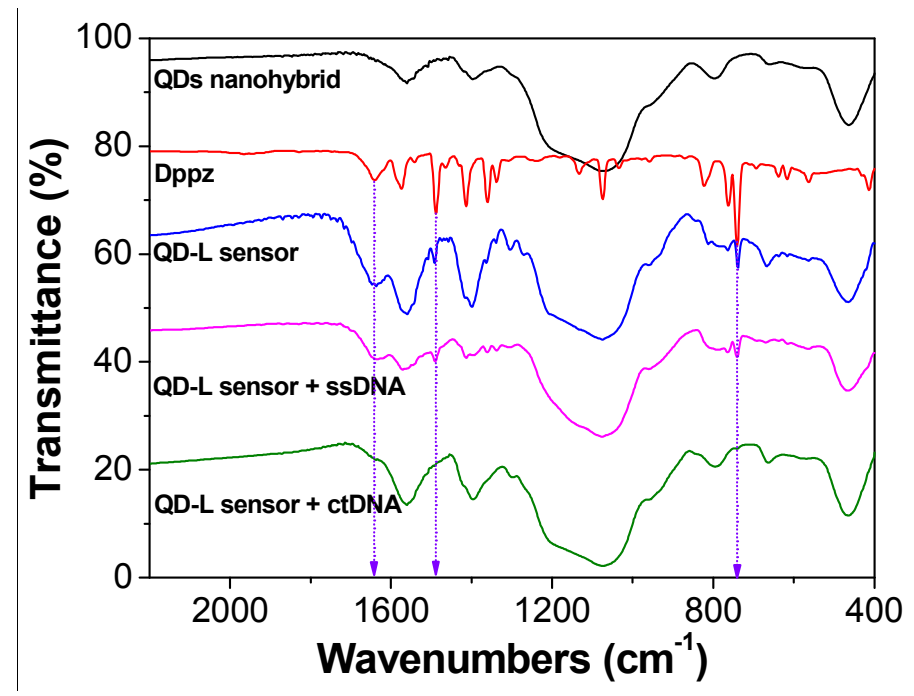

Figure S1. FT-IR spectra of the QDs nanohybrid, dppz, QD-L sensor in the absence/presence of $30 \mathrm{mg} / \mathrm{L}$ ssDNA or ctDNA.
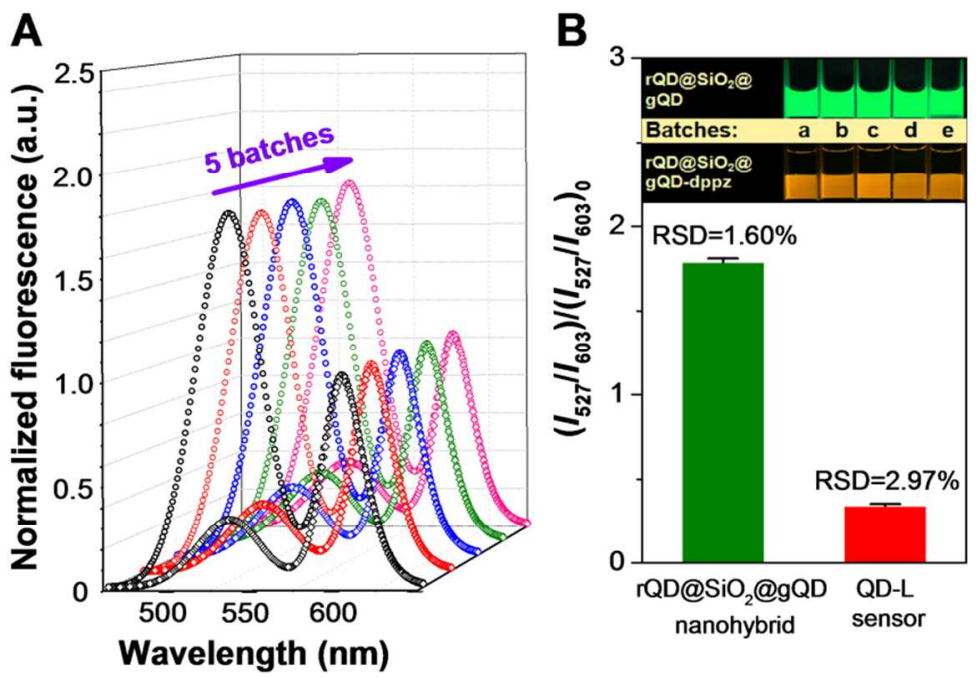

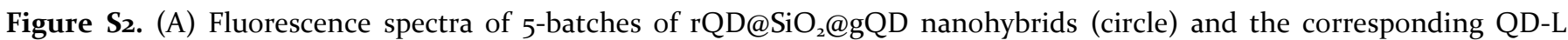
sensors (diamond). (B) Relative emission intensity ratio of the QDs nanohybrid and the QD-L sensor. The inset shows fluorescent images of the QDs nanohybrid and the QD-L sensor for 5 batches (a-e). 


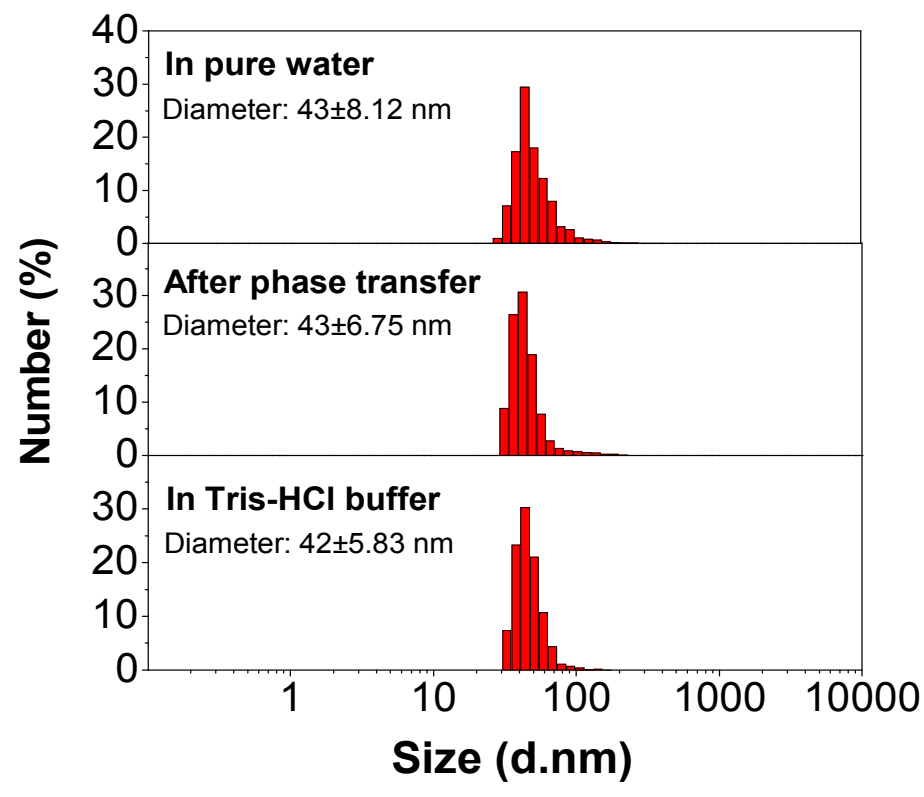

Figure S3. DLS measurements showing size distribution of rQD@SiO $@ g Q D$ nanoparticles in pure water, after phase transfer and in Tris- $\mathrm{HCl}$ buffer.



Figure S4. Ratiometric fluorescent response of the QD-dye ionic conjugate as well as the QD-L sensor versus time at different ctDNA concentrations. 

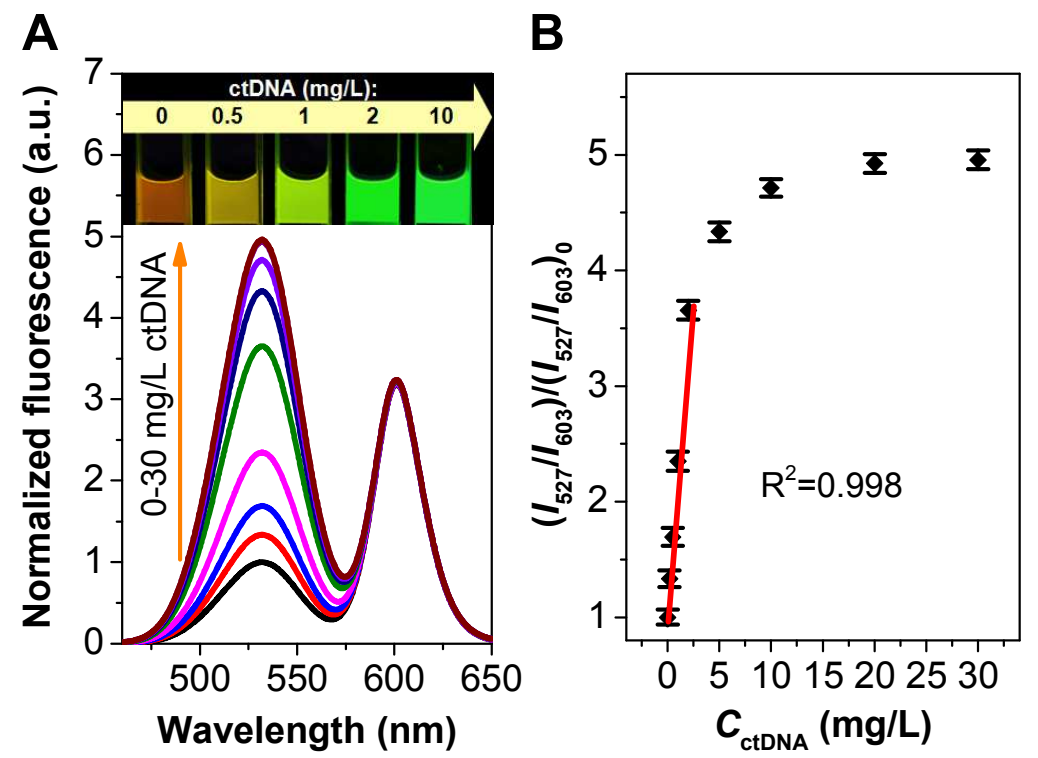

Figure S5. (A) Fluorescence spectra of the rQD@SiO ${ }_{2} @ g Q D:\left[R u(b p y)_{2}(\mathrm{dppz})\right]^{2+}$ sensor in the presence of o, o.2, o.5, 1, 2, 5, 10, $30 \mathrm{mg} / \mathrm{L}$ ctDNA. The inset shows fluorescent images of this sensor at different concentrations of ctDNA. (B) Evolution of relative emission intensity ratio of the $\mathrm{rQD}_{\mathrm{SSiO}} @ \mathrm{gQD}:\left[\mathrm{Ru}(\mathrm{bpy})_{2}(\mathrm{dppz})\right]^{2+}$ sensor with respect to the ctDNA concentration.

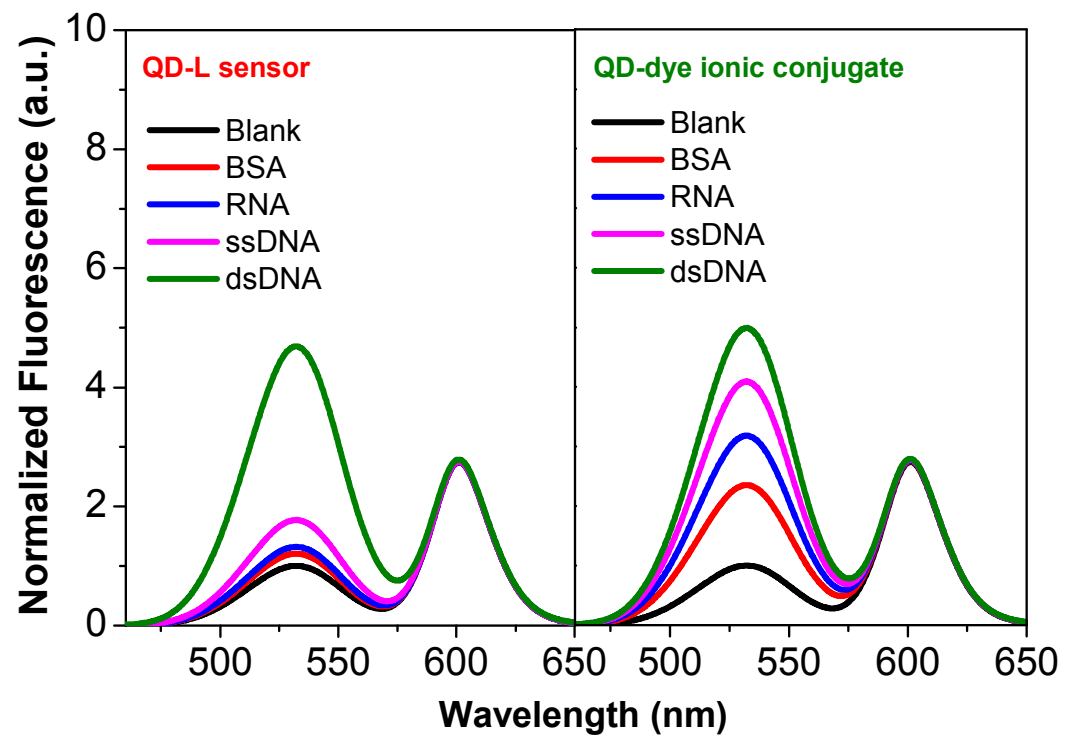

Figure S6. Fluorescence spectra of the QD-L sensor and the QD-dye ionic conjugate in the presence of different biomolecules. 
A

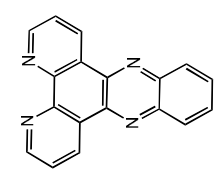

dppz

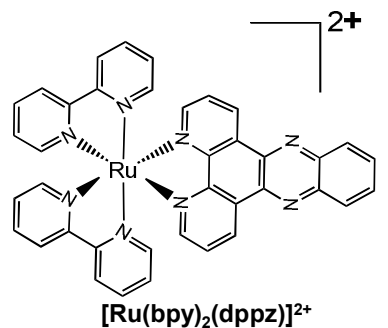

B

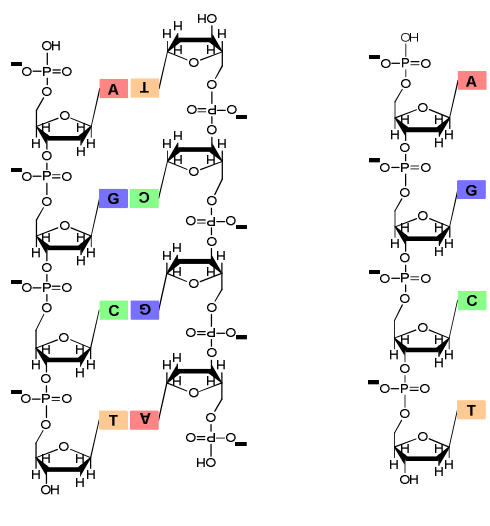

dsDNA

ssDNA

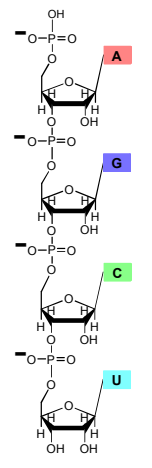

RNA

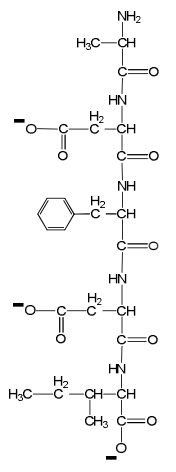

BSA

Figure $S_{7}$. Molecular structures of $(\mathrm{A}) \mathrm{dppz}$ and $\left[\mathrm{Ru}(\mathrm{bpy})_{2}(\mathrm{dppz})\right]^{2+}$, and (B) dsDNA, ssDNA, RNA and BSA.

A
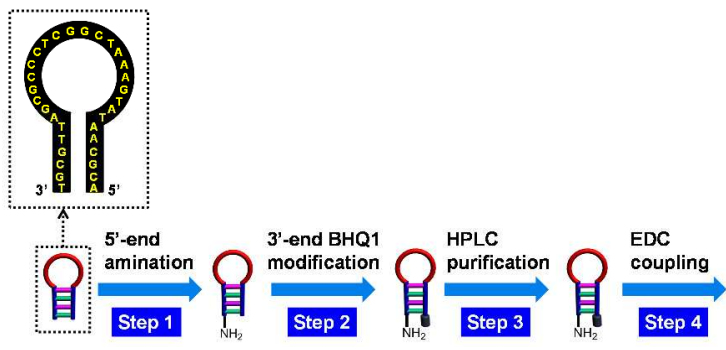

Step 4

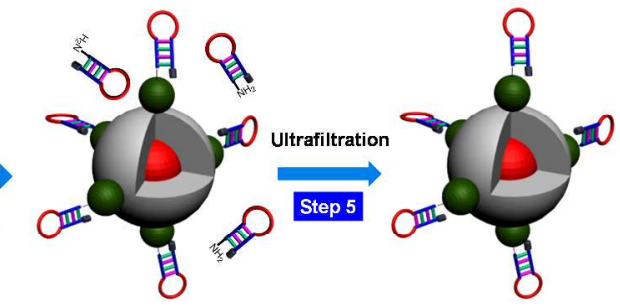

B

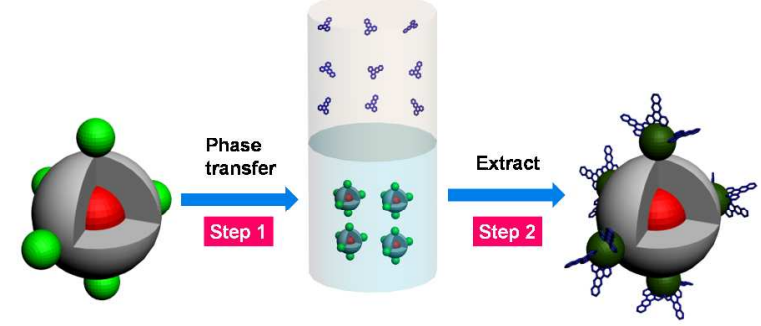

Figure S8. Comparison of fabrication process between the QD-MB sensor and the QD-L sensor. 



Figure S9. (A) Fluorescence spectra of the QD-MB sensor upon hybridizing with $0,0.2,0.5,1,2,3$, 4, and $5 \mu \mathrm{M}$ WT. (B) Fluorescence spectra of the QD-L sensor in the presence of the capture DNA $(5 \mu \mathrm{M})$ upon hybridizing with o, o.2, o.5, 1, 2, 3, 4, and $5 \mu \mathrm{M}$ WT. (C) Evolution of relative emission intensity ratio of the sensors with respect to the concentrations of WT. The inset shows fluorescent images of the QD-L sensor and the QD-MB sensor at different WT concentrations, respectively.

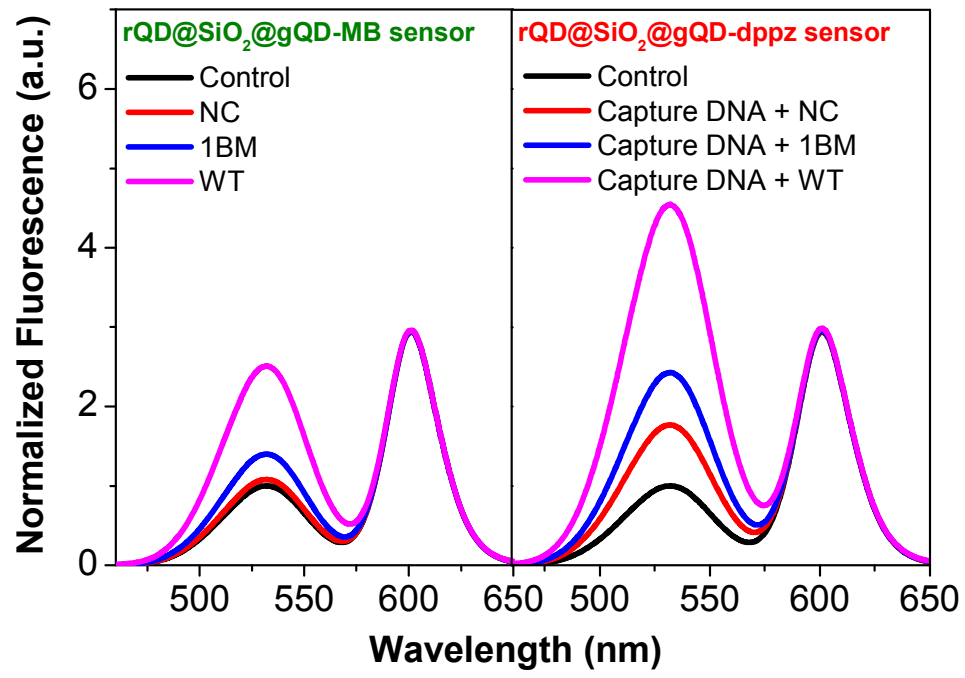

Figure S1o. Fluorescence spectra of the QD-L sensor (in the presence of $3 \mu \mathrm{M}$ capture DNA) and the QD-MB sensor upon hybridizing with $3 \mu \mathrm{M} \mathrm{NC}, 1 \mathrm{BM}$ and WT. 